\title{
Clinical features of diabetes mellitus cases complicated by Burkholderia pseudomallei septicemia
}

\author{
H.B. Quan, T.Y. Li, Y.Y. Gao and D.X. Chen \\ Department of Endocrinology, Hainan Province People's Hospital, \\ Haikou, Hainan \\ Corresponding author: H.B. Quan \\ E-mail: huibiaoquan@126.com
}

Genet. Mol. Res. 13 (2): 3108-3116 (2014)

Received March 13, 2013

Accepted October 30, 2013

Published April 17, 2014

DOI http://dx.doi.org/10.4238/2014.April.17.7

\begin{abstract}
The aim of this study was to analyze the clinical characteristics of diabetes mellitus patients with Burkholderia pseudomallei septicemia and evaluate strategies of diagnosis and treatment. The clinical characteristics, diagnosis, treatment, and prognosis of 39 diabetes mellitus patients with B. pseudomallei septicemia were retrospectively analyzed. Farmers, fishermen and workers were found to be high-risk groups. The clinical manifestations of patients were diverse without specific features, but mainly presented manifestations of acute fulminant septicemia, diabetic ketoacidosis, and abscesses in tissues or/and organs. Patients showed high mortality and misdiagnosis rates and were prone to relapses and long treatment duration as there are currently few effective and sensitive antibiotics for the disease. Consequently, the cost of treatment for the disease was high. Early diagnosis, a prolonged course of heavy doses of sensitive intravenous antibiotics, drainage of abscesses, intensive insulin therapy, and supportive treatment are the keys for successful management of the disease. Regular follow-ups combined with long-
\end{abstract}


term blood glucose control can help reduce the disease recurrence.

Key words: Type 2 diabetes mellitus; Burkholderia pseudomallei; Septicemia

\section{INTRODUCTION}

Endemic zoonosis induced by Burkholderia pseudomallei (BP), a Gram-negative aerobacillus, is defined as meliodosis (MD), which mainly occurs in tropical and subtropical areas with high morbidity in Thailand, Malaysia, India, and Australia (Lee et al., 2009; Saravu et al., 2010). MD has been found in Hainan, Guangdong, Guangxi, and Taiwan, with Hainan identified as the main epidemic focus of the disease, as well as the area with the highest separation rate of BP. BP seriously threatens the health of residents in the epidemic focus, and BP septicemia primarily occurs in populations with low-immune function, e.g., patients with diabetes are particularly susceptible to MD (Hassan et al., 2010; Vidyalakshmi et al., 2012). Diabetes complicated by BP septicemia is dangerous for patients, as it cannot currently be treated effectively with conventional antibiotics. Moreover, its misdiagnosis and mortality rates are high, and consequently, so are its associated medical expenses.

The epidemiological history of MD is very important to diagnosis and treatment; diagnosis of MD is difficult in non-epidemic areas, and it is often misdiagnosed if attention is not paid to recent history of travelling to an affected area. An increasing number of migrant workers and travelers have arrived in Hainan since it became an international tourism island; thus, the development of tourism and agriculture has enhanced the opportunity for MD to spread to other places, which has required attention to be paid to the health of migrant workers and travelers (Shibuya et al., 2007). Therefore, clinical doctors in most non-affected areas as well as in the epidemic focus must heighten their vigilance against MD and increase their recognition ability of this disease. In this study, 39 patients with diabetes complicated by BP septicemia who were treated in our hospital between October 2002 and March 2012 were retrospectively analyzed and followed up to explore their clinical characteristics, diagnosis, treatment, and outcomes.

\section{MATERIAL AND METHODS}

\section{Study subjects}

The clinical characteristics, diagnosis, treatment, and outcomes of 39 patients (32 males, 7 females; age range, 18-69 years; average age, $49.7 \pm 5.6$ years) with diabetes complicated with BP septicemia were analyzed, including 35 patients with type II diabetes and four with type I diabetes. Twenty-three of the 39 patients were farmers, seven were fishermen, and five were workers. Six other patients relapsed and were readmitted to the hospital, raising the total to 45 cases, which were all diagnosed with BP septicemia due to the pure growth of BP in their blood cultures. In addition, BP was isolated from two cases of bone marrow culture, six cases of sputum culture, and five cases of pus culture. The average time from admission to hospital to diagnosis was 7.1 \pm 2.3 days (range: 5-12 days), and the average length of stay was $25.3 \pm 15.7$ days (range: 4-66 days). Four patients died before diagnosis. 


\section{Diagnosis}

For diagnosis, blood cultures were performed three times for all patients admitted to the hospital, as well as bone marrow, pus, or other body fluid bacterial cultures if necessary. Blood cultures were cultivated in a standard aerobic culture bottle obtained from BD (USA). Bacteria were isolated with 5\% sheep blood agar prepared by Columbia Agar. An automated system for identification and susceptibility tests (BioMerienx, France) was utilized to identify the bacteria, and susceptibility tests were performed according to the broth microdilution method recommended by the National Committee for Clinical Laboratory Standards (NCCLS), and the corresponding results were evaluated according to NCCLS criteria (Table 1).

\section{Efficacy estimation}

Treatment efficacy was classified into five categories: clinical recovery, improved, not improved, deteriorated, and dead. Clinical recovery was determined when symptoms in patients disappeared, treatment of susceptible antibiotics was performed for at least 4 weeks, body temperature was normal for more than 2 weeks, and the blood culture results were negative. The improved condition was determined when the symptoms in patients improved significantly, the treatment of susceptible antibiotics was performed for at least 2 weeks, body temperature was normal for more than 1 week, and/or the results of blood culture were negative. The not improved condition was determined when no susceptible antibiotic was administrated in patients or when the treatment was shorter than 1 week and body temperature did not return to normal. The deteriorated condition was determined when no susceptible antibiotic was administrated in patients and their symptoms and conditions deteriorated. All patients were followed up post-discharge.

\section{Statistical analysis}

The $t$-test was used to compare groups and the chi-square test was used to evaluate rates.

\section{RESULTS}

All cases were misdiagnosed before isolating BP from blood cultures. There were 14 cases diagnosed as lung infections or tuberculosis, 13 cases of ordinary bacterial sepsis, 6 cases of urinary infection, and 1 case of each of the following diseases: suppurative arthritis, infective endocarditis, scrub typhus, leptospirosis, liver abscess, and jaundice hepatitis.

\section{Clinical manifestations}

The main clinical manifestations and laboratory data of these cases are summarized in Table 1. All patients were characterized by shivering and high fever; continuous fever presented in patients with a maximum of $42^{\circ} \mathrm{C}$. Thirty-five cases were complicated with abscesses; three sites of abscess simultaneously occurred in two cases, and two sites of abscess were found in six cases. The mean fasting blood-glucose and HbAlc levels were $16.7 \pm 4.9$ $\mathrm{mM}$ (range: $10.7-29.1 \mathrm{mM}$ ) and $12.6 \pm 2.4 \%$ (range: $9.8-15.3 \%$ ), respectively. 


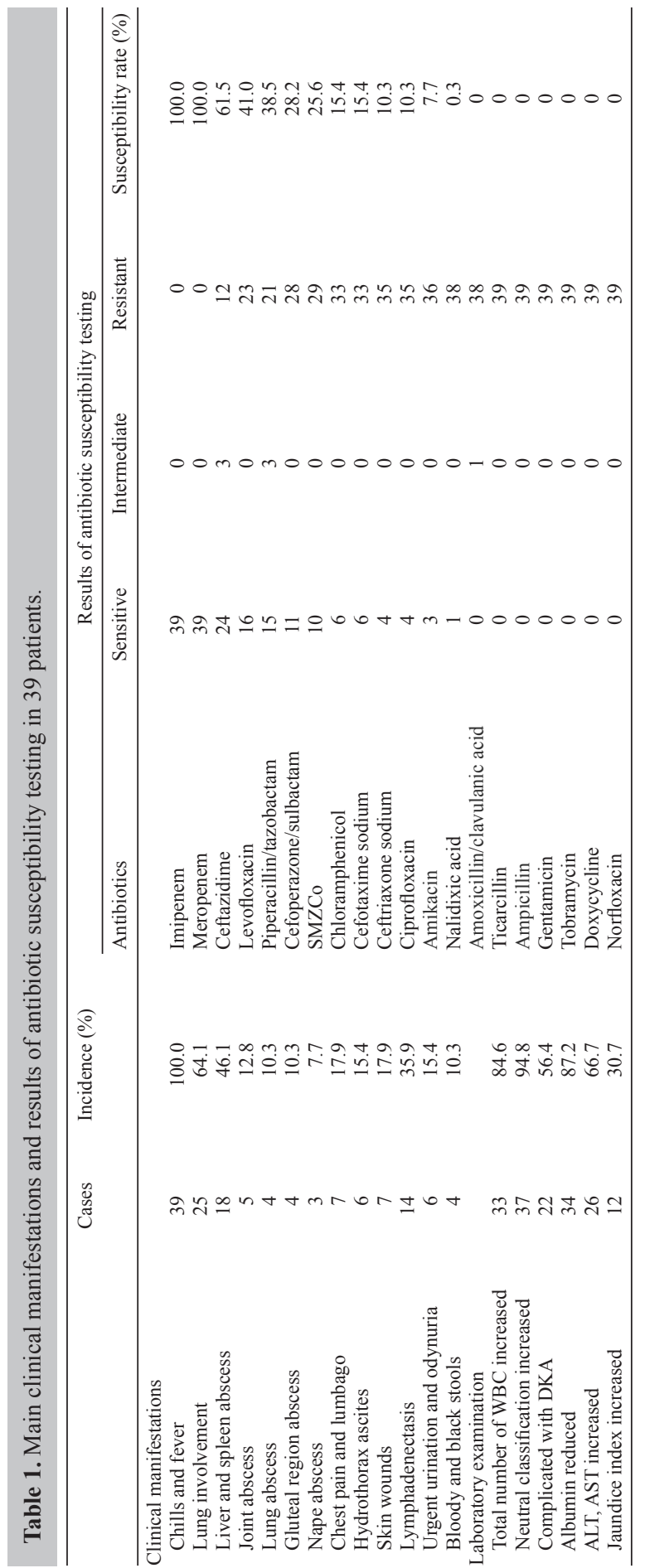




\section{Diagnosis, treatment, outcomes, and follow-up}

All patients admitted to the hospital were given anti-infection therapy and blood glucose control by insulin. There were 22 cases complicated with diabetic ketoacidosis (DKA), five cases with multiple organ failure, fives cases with septic shock, and six cases with double infection (one case occurred before diagnosis and five cases occurred during treatment).

After clinical treatment, 17 patients recovered, accounting for $43.6 \%$ (including the 6 relapsed cases). Imipenem was used in 10 cases, ceftazidime was used in three cases, piperacillin/tazobactam plus levofloxacin was used in two cases, piperacillin/tazobactam plus amikacin was used in one case, and cefotaxime sodium was used in one case. In one case, the patient was primarily complicated by lung MD, and BP was also isolated from blood and sputum cultures. This patient was considered to be cured as imipenem treatment was administrated for 29 days, the body temperature remained normal for 17 days, and the blood culture turned negative. However, the pulmonary foci of this patient relapsed 3 months after being cured, and became complicated by a large, ulcerating buttock abscess. After readmission to the hospital, BP was isolated from pus and blood cultures, which was only sensitive to imipenem. Thus, the abscess and pulmonary foci were treated with imipenem for 63 days by systematic therapy. In another relapsed case, the patient was primarily complicated with a right groin abscess and hepatapostema, and BP was isolated from both pus cultures. The pus of the right groin abscess was obtained by abscess incision, whereas the hemorrhagic pus was extracted after impalement. The patient was administrated with cefotaxime sodium for 16 days, their body temperature remained normal for 16 days, the right groin abscess was cured, and the hepatapostema shrunk based on review by ultrasound. The cefotaxime sodium treatment was performed for 7 days after the patient was discharged as they refused liver puncture or surgery. This case relapsed 2 months after being discharged. After readmission to the hospital, there were no abnormalities found in review by ultrasound, and the patient was cured after 28 days of cefotaxime sodium treatment. The report of susceptibility tests revealed that one case was sensitive to ceftazidime, but showed no clinical effects in treatment, and the patient was cured when imipenem was administrated. In one case complicated with a splenic abscess, the body temperature remained high after 1 week of imipenem administration. The review by ultrasound indicated that the abscess had expanded, and therefore surgery was performed to remove the spleen. After surgery, BP could be isolated from both the splenic pus and blood culture, and the patient was cured following administration of imipenem. In all of the 17 cured cases, insulin was utilized to control blood glucose levels; an insulin pump was applied in seven cases, and albumin and immunoglobulin were used simultaneously to support symptomatic treatment. We failed to follow two of these cases, and the remaining 15 patients have not relapsed so far.

Among the 12 patients $(30.8 \%)$ showing recovery, five were treated with imipenem, three with ceftazidime and levofloxacin, two with ceftazidime and SMZCo, one with piperacillin/ tazobactam plus levofloxacin, and one with piperacillin/tazobactam plus chloramphenicol. To date, eight of these patients have not relapsed and four patients were lost.

Ten diabetes patients $(25.6 \%)$ complicated with DKA died. Four of them died before diagnosis in hospital, including two cases of septic shock and multiple organ failure, and another two cases of acute respiratory distress syndrome (ARDS). The other six patients died after leaving the hospital (deterioration was found in four cases, and two patients did not recover and died within 1 month of leaving hospital). One case was sensitive to piperacillin/tazobactam, ce- 
foperazone/sulbactam, and levofloxacin, but antibiotic therapy was not effective after 2 weeks of treatment, thus imipenem was applied to the patient for 1 week, whose body temperature fluctuated between $37^{\circ}$ and $38^{\circ} \mathrm{C}$ with double infection and upper gastrointestinal hemorrhage. One patient was sensitive to cefoperazone/sulbactam, but no therapeutic effect was observed. Two patients were resistant to ceftazidime plus tinidazole. Two other patients showed negative efficacy with mezlocillin plus levofloxacin; they were only sensitive to imipenem.

\section{Medical expense}

The mean medical expense was 23,075.2 $\pm 3665.9 \mathrm{RMB} /$ case (range: $7268.4-59,642.4$ $\mathrm{RMB} /$ case). The results showed that days of blood sugar control, hospitalization days, medical expenses, and the rate of curing and improvement were better in endocrinology hospitalized patients compared to non-endocrinology patients (Table 2).

Table 2. Comparison of indicators between endocrinology hospitalized patients and non-endocrinology.

\begin{tabular}{lccccc}
\hline Department & $\mathrm{N}$ & $\begin{array}{c}\text { Days of blood } \\
\text { sugar control }\end{array}$ & $\begin{array}{c}\text { Hospitalization } \\
\text { days }\end{array}$ & $\begin{array}{c}\text { Medical costs } \\
\text { (yuan/case) }\end{array}$ & $\begin{array}{c}\text { Cases of curing and improved (N) } \\
\text { Rate of curing and improved (\%) }\end{array}$ \\
\hline Endocrinology & 18 & $4.6 \pm 1.2^{\#}$ & $23.1 \pm 10.2^{\#}$ & $18517.6 \pm 2885.8^{\#}$ & $(10+6)$ \\
Non-endocrinology & 21 & $9.3 \pm 3.4$ & $29.3 \pm 16.4$ & $25711.1 \pm 3903.4$ & $\begin{array}{c}88.9 \% \%^{\#} \\
(7+6) \\
61.9 \%\end{array}$ \\
\hline
\end{tabular}

\section{DISCUSSION}

Due to decreased immune function, patients with diabetes are vulnerable to various infections. In our study, 39 patients complicated with systemic infection were diagnosed with $\mathrm{BP}$ septicemia after pure growth of BP was isolated in their blood cultures.

MD is a zoonotic disease caused by Pseudomonas melioidosis bacillus. Humans become infected with BP mainly by direct contact of injured skin with polluted soil, sewage, or crops (Tay et al., 2012); it may also result from consuming water or food contaminated with BP or by breathing pollutants that contain the bacteria. In addition, some infections are induced by close contact among people and by bites from hematophagus insects (e.g., fleas, mosquitoes). The latent period of BP infection varies greatly, and ranges from 2 days to 20 years with a typical period of 4-5 days. Farmers and fishermen were found to be particularly susceptible to BP $(30 / 39,76.9 \%)$, which is consistent with published reports (Hassan et al., 2010).

Clinical characteristics of MD vary, which can manifest as septicemia, septic shock, or subclinical infection. The mortality of patients with acute septicemia was reported as 4065\% (Lee et al., 2009; Deris et al., 2010; Hassan et al., 2010; Vidyalakshmi et al., 2012). In recent years, the mortality rate has reduced owing to improvements in knowledge about septicemia and the application of antibiotics. Diabetic patients infected with BP can easily develop septicemia. Puthucheary et al. (1992) reported that the mortality rate of 50 patients with BP septicemia was $65 \%$, and $76 \%$ of patients had underlying basic diseases, among which diabetes was the most common; the majority of the patients displayed multiple organ-affected symptoms with a $58 \%$ rate of lung involvement. In our study, lung involvement accounted for $68.2 \%$ of cases, which basically accorded with the literature (Deris et al., 2010). Ten patients 
died among the 39 patients with acute septicemia, resulting in a mortality rate of $25.6 \%$ in this study. Diabetic patients complicated with BP septicemia always develop DKA and abscesses, which is very dangerous for patients and is associated with poor prognosis, and serious complications, including septic shock, ARDS, and multiple organ failure, will present quickly, and even cause death if patients are not treated timely and effectively (Puthucheary, 2009).

The key for treating diabetes combined with BP septicemia lies in the timely selection of adequate sensitive antibiotics and strengthening blood glucose control. Ceftazidime has been recommended as the drug of choice for BP septicemia (Puthucheary et al., 2001). However, in this study, the susceptibility rate of ceftazidime was only $61.5 \%$, and one patient who was sensitive to ceftazidime, as indicated by the susceptibility test report, did not benefit from the treatment, which demonstrated that a portion of BP was resistant to ceftazidime in this region, and that conventional concentrations of sensitive drugs may not always be effective. Therefore, doctors should not only rely on the report of the susceptibility test to treat BP septicemia, but should also closely observe patients' conditions and adjust the therapy accordingly. All 39 patients in our study were sensitive to imipenem, which could be applied in deescalation therapy if the patient's condition becomes very dangerous. Surgical drainage treatment should be conducted in a timely manner in patients with festering lesions. With respect to abscess cases that are difficult to treat medically, such as splenic abscess, surgical resection of diseased organs can be employed (Ng et al., 2008; Shih et al., 2009) to improve the cure rate. For diabetic patients with BP septicemia, good control of blood glucose levels can improve the curing rate, decrease hospitalization days, reduce the administration of antibiotics, and reduce medical costs. In addition, diabetic patients with BP septicemia have reduced cellular and humoral immunity function; thus, attention should be paid to supporting treatments along with the application of susceptible antibiotics to correct hypoalbuminemia, increase the immunity function with immunoglobulin, and decrease the double-infection, which will greatly improve the rescue success rate and reduce mortality rates.

BP septicemia is characterized by high mortality, a long treatment cycle, and high expenses; timely diagnosis and effective therapy are the most useful measures to reduce medical expenses and mortality. In our study, the patients stayed in hospital for an average of $25.3 \pm 15.7$ days (range: 4-66 days), which was consistent with the literature (Pande and Kadir, 2011). Due to the complex clinical manifestations of BP septicemia, the misdiagnosis rate was $100 \%$, which also resulted from the fact that BP was easily confused with other diseases or Pseudomonas. Dharakul et al. (1997) suggested that high mortality correlates with the delay of pathogen isolation and identification. Therefore, an advanced identification system would enhance the ability for identification and diagnosis of the bacteria in the laboratory. The BioMerienx VITEK-32 automatic identification system utilized in our hospital has the highest separation rate of BP in China at present, whereas reports from other countries have recommended the NFT and XID32 systems with accuracy of more than $99 \%$. If patients who are in an affected area or who have had contact with the epidemic focus develop an unexplained fever or suppurative diseases, especially with multiple abscesses, then BP septicemia should be considered, and cultures of blood, pus, and/or bone marrow should be performed to identify pathogenic bacteria. During the bacterial culture of suspicious patients, clinicians should keep in touch with the bacterial culture laboratory every day rather than waiting, so as to be able to diagnose the patients as soon as possible.

There are various therapeutic schedules possible for MD; thus, suitable antibiotics and therapy should be determined according to the clinical condition of individual patients. 
Since BP is so tenacious that it cannot be eliminated completely and is easy to relapse, adequate doses of susceptible antibiotics and treatment periods are required for effective therapy. Susceptible antibiotics should be administrated intravenously for 3-4 weeks to treat BP septicemia, and oral SMZCo can be taken simultaneously for 6 months to avoid relapse in patients with pyogenic lesions (Subbalaxmi et al., 2011). Moreover, effective, long-term glycemic control is an important measure for diabetics to improve immune function, reduce infection, and prevent the relapse of disease.

MD is preventable (Limmathurotsakul and Peacock, 2011). The susceptible population includes farmers, fishermen, and workers, whose education levels and incomes are relatively low. MD with BP septicemia is disastrous for patients and their families. Studies have indicated that MD is more prevalent in the rainy season (Christenson et al., 2003; Ezzedine et al., 2007). Therefore, medical and epidemic prevention staff should gain more knowledge of this disease and engage in more public outreach and education work in the susceptible population in epidemic areas. The chances of BP infection can be reduced remarkably in people in epidemic areas, who can be well protected before making contact with potentially contaminated soil and water sources (Kung et al., 2013). In recent years, some melioidosis vaccine studies have been reported (Easton et al., 2011; Lassaux et al., 2013), and we look forward to further achievements in this field. The successful development of a melioidosis vaccine showing ideal clinical effects would significantly decrease the incidence of melioidosis sepsis.

\section{REFERENCES}

Christenson B, Fuxench Z, Morales JA, Suarez-Villamil RA, et al. (2003). Severe community-acquired pneumonia and sepsis caused by Burkholderia pseudomallei associated with flooding in Puerto Rico. Bol. Asoc. Med. P. R. 95: 17-20.

Deris ZZ, Hasan H and Siti Suraiya MN (2010). Clinical characteristics and outcomes of bacteraemic melioidosis in a teaching hospital in a northeastern state of Malaysia: a five-year review. J. Infect. Dev. Ctries. 4: 430-435.

Dharakul T, Songsivilai S, Anuntagool N, Chaowagul W, et al. (1997). Diagnostic value of an antibody enzyme-linked immunosorbent assay using affinity-purified antigen in an area endemic for melioidosis. Am. J. Trop. Med. Hyg. 56: 418-423.

Easton A, Haque A, Chu K, Patel N, et al. (2011). Combining vaccination and postexposure CpG therapy provides optimal protection against lethal sepsis in a biodefense model of human melioidosis. J. Infect. Dis. 204: 636-644.

Ezzedine K, Malvy D, Steels E, De Dobbeeler G, et al. (2007). Imported melioidosis with an isolated cutaneous presentation in a 90-year-old traveller from Bangladesh. Bull. Soc. Pathol. Exot. 100: 22-25.

Hassan MR, Pani SP, Peng NP, Voralu K, et al. (2010). Incidence, risk factors and clinical epidemiology of melioidosis: a complex socio-ecological emerging infectious disease in the Alor Setar region of Kedah, Malaysia. BMC Infect. Dis. 10: 302 .

Kung CT, Li CJ, Ko SF and Lee CH (2013). A melioidosis patient presenting with brainstem signs in the emergency department. J. Emerg. Med. 44: e9-12.

Lassaux P, Peri C, Ferrer-Navarro M, Gourlay LJ, et al. (2013). A structure-based strategy for epitope discovery in Burkholderia pseudomallei OppA antigen. Structure 21: 167-175.

Lee HM, Choi SH, Chung JW, Ahn J, et al. (2009). A case of disseminated melioidosis in a migrant worker from Thailand. Korean J. Lab. Med. 29: 140-144.

Limmathurotsakul D and Peacock SJ (2011). Melioidosis: a clinical overview. Br. Med. Bull. 99: 125-139.

Ng CY, Leong EC and Chng HC (2008). Ten-year series of splenic abscesses in a general hospital in Singapore. Ann. Acad. Med. Singapore 37: 749-752.

Pande KC and Kadir KA (2011). Melioidosis of the extremities in Brunei Darussalam. Singapore Med. J. 52: 346-350.

Puthucheary SD (2009). Melioidosis in Malaysia. Med. J. Malaysia 64: 266-274.

Puthucheary SD, Parasakthi N and Lee MK (1992). Septicaemic melioidosis: a review of 50 cases from Malaysia. Trans. R. Soc. Trop. Med. Hyg. 86: 683-685.

Puthucheary SD, Vadivelu J, Wong KT and Ong GS (2001). Acute respiratory failure in melioidosis. Singapore Med. J. 42: $117-121$. 
Saravu K, Mukhopadhyay C, Vishwanath S, Valsalan R, et al. (2010). Melioidosis in southern India: epidemiological and clinical profile. Southeast Asian J. Trop. Med. Public Health 41: 401-409.

Shibuya H, Taniguchi Y, Tashiro N, Hara K, et al. (2007). A Japanese case of melioidosis presenting as multiple organ lesions accompanied by sepsis and disseminated intravascular coagulation, after a visit to Thailand. Kansenshogaku Zasshi 81: 297-301.

Shih HI, Chuang YC, Cheung BM, Yan JJ, et al. (2009). Sporadic and outbreak cases of melioidosis in southern Taiwan: clinical features and antimicrobial susceptibility. Infection 37: 9-15.

Subbalaxmi MV, Chandra N, Rao MN, Vemu L, et al. (2011). Burkholderia pseudomallei: an uncommon cause of bacteraemic pneumonia in a diabetic. Indian J. Chest Dis. Allied Sci. 53: 185-187.

Tay TF, Maheran M, Too SL, Hasidah MS, et al. (2012). Glycogen synthase kinase-3ß inhibition improved survivability of mice infected with Burkholderia pseudomallei. Trop Biomed. 29: 551-567.

Vidyalakshmi K, Lipika S, Vishal S, Damodar S, et al. (2012). Emerging clinico-epidemiological trends in melioidosis: analysis of 95 cases from western coastal India. Int. J. Infect. Dis. 16: e491-e497. 